\title{
Point-of-care haemostatic management in orthotopic liver transplantation of a patient with a coronary stent
}

\section{Katsanoulas K., Katsika E, Markopoulos I., Georgopoulou E., Bilali P., Vranas D. Hippokrateion General Hospital, Anaesthesiology Dept., Thessaloniki, Greece}

\section{BACKGROUND}

Challenges in Orthotopic Liver Transplantation(OLT)

$>$ Distinct phases with specific physiological alterations and major hemodynamic fluctuations.

$>$ Transfusion more often refers to blood products than RBCs.

$>$ Coagulation defects, conventionally determined, do not predict blood product requirements.

$>$ OLT without intraoperative transfusions showed better early outcome; preemptive FFP transfusion should be avoided.

\section{Challenges in haemostatic management in OLT}

$>$ Reduced levels of both procoagulant factors and inhibitors.

$>$ Thrombocytopenia/dysfunction is counterbalanced by the increased levels of fVIII and vWf.

Hyperfibrinolysis is a major issue after reperfusion.

ESA 2013: "We recommend the use of perioperative ROTEM/TEG monitoring for targeted management of coagulopathy".

$>$ Goal-directed POC parameters algorithmic approach.

ROTEM-based clot strength showed hypocoagulation correlated to the level of liver impairment,

but

$>$ TGT (thrombin generation test) indicated a preserved or increased thrombin release suggesting even a potential for hypercoagulability.

$>$ ROTEM may not be appropriate for haemostasis assessment in patients with cirrhosis and may lead to unnecessary coagulation factors transfusion (!)

\section{CASE REPORT}

* Male, 66 y, wt 78kg, ht $173 \mathrm{~cm}$

- NASH Cirrhosis MELD score 17

* Four HE episodes, type II diabetes

* Coagulation profile : PT 16/11.2, APTT 39.5/31, FIB $2.43 \mathrm{~g} / \mathrm{dl}$, PLTs $134000 \mu / 1$

* ICH, PTCA (LAD stent) 1 y ago, PAPs $68 \mathrm{~mm}$, EF 60\%,

* Aspirin, clopidogrel, diuretics, $\beta$-blocker, insulin, prazole

Orthotopic liver transplantation (piggy pack technique)

* Hbg 9.9 (start) - 9.3 (end) g/dl

* 3 PRBCs after reperfusion at Hbg 6.6 g/dl

* Fluids balance $+5150 \mathrm{ml}$

* Anhepatic phase 2.5 hrs, uneventful reperfusion

* Total OLTx duration 8 hrs.

* Acceptable haemodynamics CI 3.1-4.8, PVRI 60-96,

$$
\mathrm{Ca}-\mathrm{vO}_{2} \text { 2.9-4.2, } \mathrm{SvO}_{2} 85-76 \%
$$

* ICU, 2 d mechanical ventilation.

* 1 mo in ward, chest pain episode, transient and improving CNS impairment.

* Satisfactory graft function, discharged 1 month later.

REFERENCES

- Kirchner C. Coagulation management with factor concentrates in liver transplantation:a single-center experience.Transfusion 2014;54:2760-8 - Lentschener C. Assessment of haemostasis in patients with cirrhosis:Relevance of the ROTEM tests? Eur J Anaesthesiol 2016; 33:126-133

- Herbstreit F. Monitoring of haemostasis in liver transplantation: comparison of laboratory based and point of care tests. Anaesthesia. 2010;65(1):44-9

\section{DISCUSSION}

Haemostatic management and transfusions were directed by ROTEM ${ }^{\circledR}$ following a published algorithm.(Goerlinger K, 2006, rev.2014). PLT aggregometry was not available.

We compared standard haemostatic lab tests data (SLT) to ROTEM data, suggesting agreements and disagreements on patient's haemostatic state interpretation.
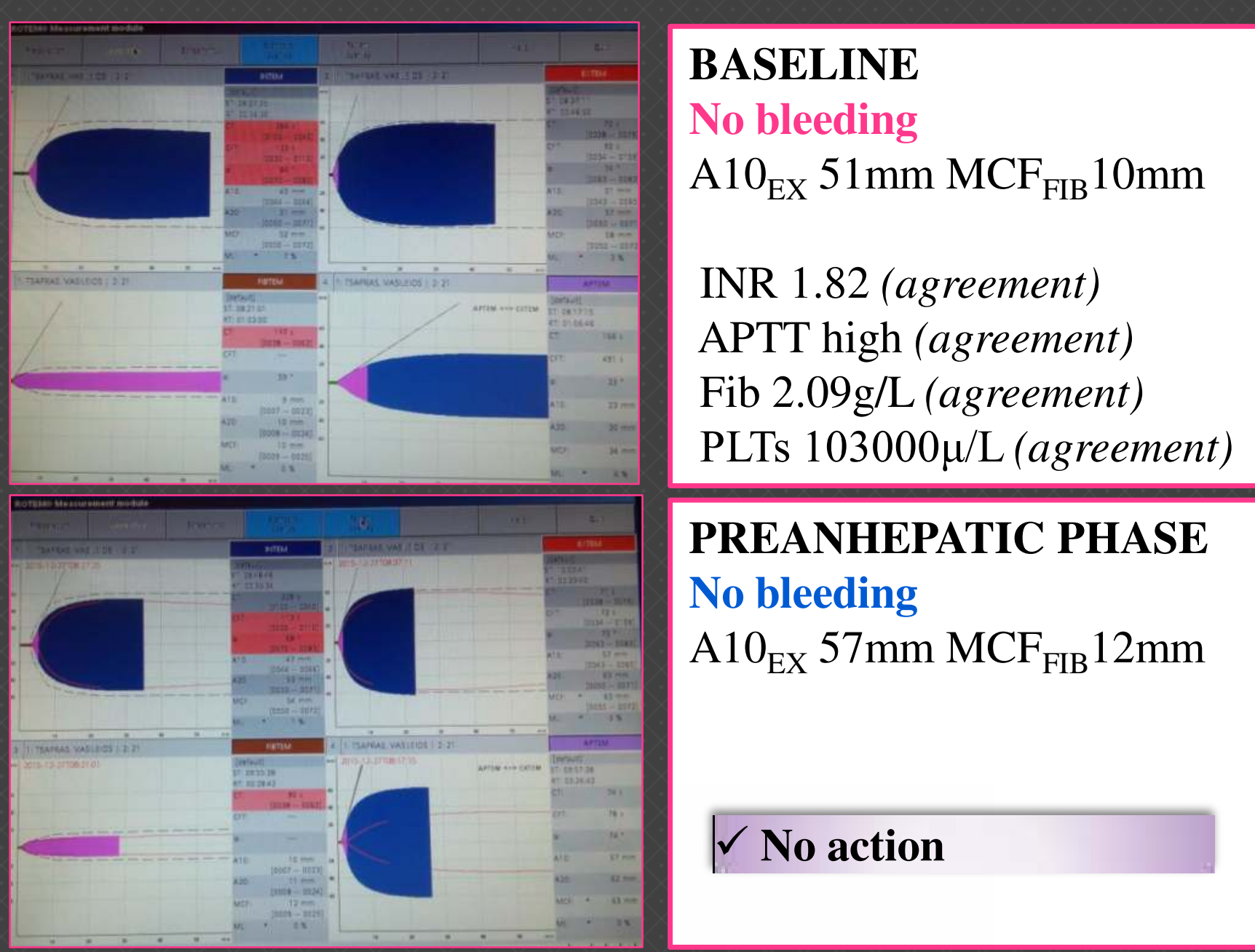

PREANHEPATIC PHASE

No bleeding

$\mathrm{A} 10_{\mathrm{EX}} 57 \mathrm{~mm} \mathrm{MCF} \mathrm{FIB}_{12} \mathrm{~mm}$

\section{$\checkmark$ No action}

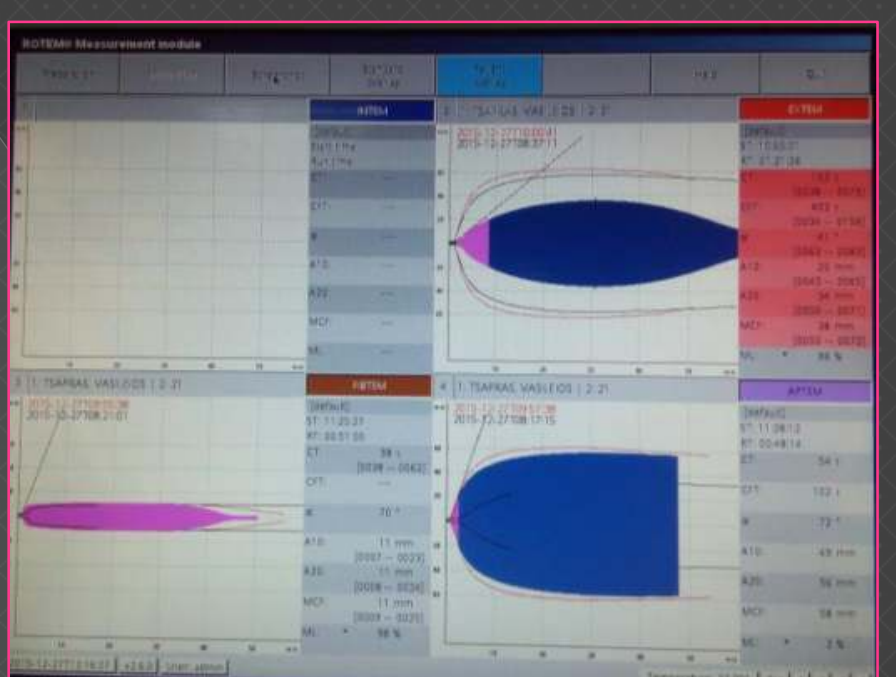

\section{ANHEPATIC PHASE}

Mild bleeding

$\mathrm{CT}_{\mathrm{EX}} \mathbf{1 0 3}_{\mathrm{sec}} \mathrm{CFT}_{\mathrm{EX}} \mathbf{4 0 3} \mathrm{sec}$

$\mathrm{A} 10_{\mathrm{EX}} \mathbf{2 5} \mathrm{sec} \mathrm{MCF}_{\mathrm{FIB}} 11 \mathrm{sec}$

INR 2.07 (agreement)

APTT $138.4 \mathrm{sec}$ (agreement)

Fib $1.35 \mathrm{~g} / \mathrm{L}$ (disagreement)

PLTs $148000 \mu / \mathrm{L}($ dis/ment $)$

\section{TRANSFUSION: 1 g TXA, 3 units FFP, 6 units Platelets}

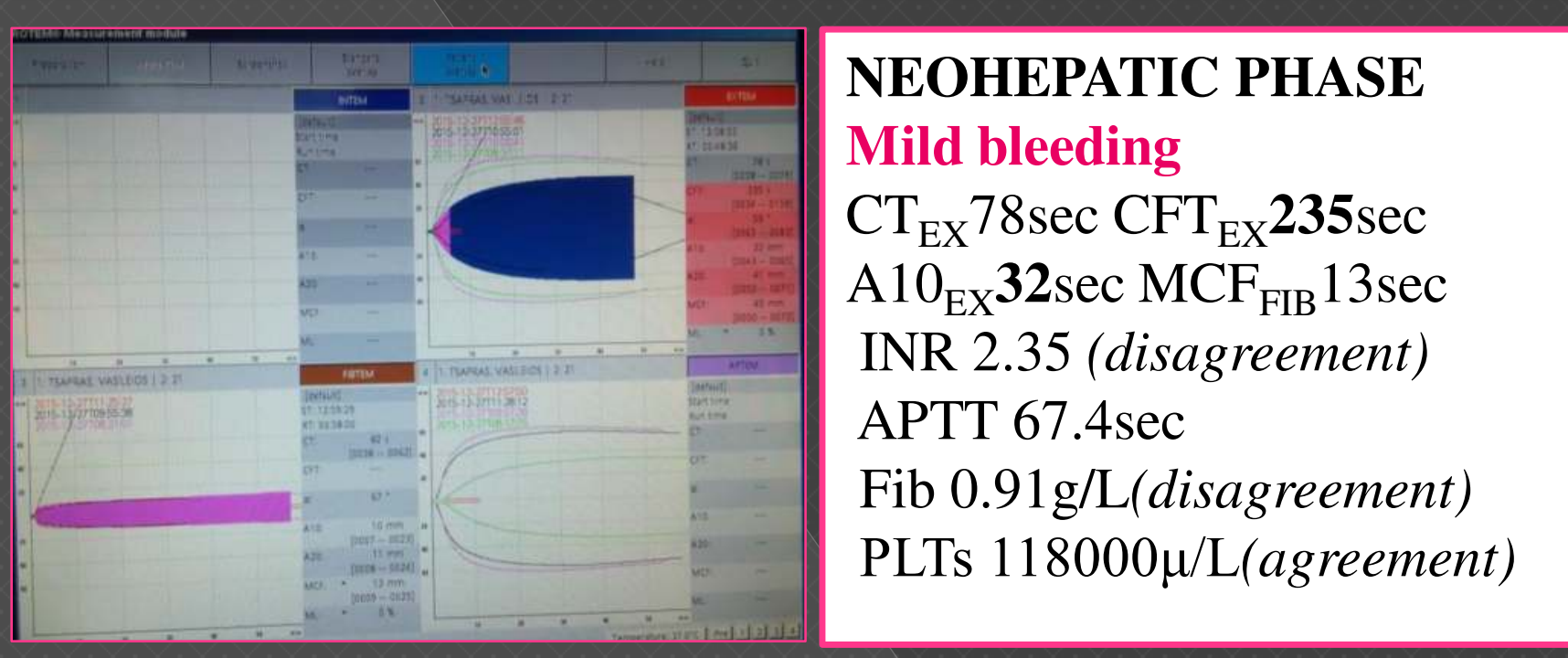

$\checkmark$ No PLTs transfused (although considered)

$\checkmark$ PLT related hypercoagulative danger/ increased mortality $\checkmark$ Coronary stent in place

$\checkmark$ TRANSFUSION: 10 units cryoprecipitate (to enhance clot strength, counterbalancing low PLTs count)

$\checkmark$ Bleeding ceased completely

\section{LEARNING POINTS}

Intraoperative standard lab tests during OLT may lead to improper even dangerous decisions especially in the presence of co-morbidities affecting haemostasis.

$\square$ Point-of-care haemostatic management along with close surgical bleeding evaluation contributed to successful decision making in the management of this complex case. 